VOLUMEN 38 | N 3 | DICIEMBRE 2020

FECHA DE RECEPCIÓN: 21/07/2020 FECHA APROBACIÓN: 24/02/2021 FECHA DE PUBLICACIÓN: 06/04/2021

1. Economista en libre ejercicio. Cuenca - Ecuador

2. Universidad de Cuenca. Facultad de Ciencias Económicas y Administrativas- FCEA. Carrera de Administración de Empresas Dual.

Cuenca - Ecuador

3. Universidad de Cuenca. Facultad de Ciencias Económicas y Administrativas - FCEA. Carrera de Administración de Empresas. Azogues - Ecuador

\begin{tabular}{c|c} 
Revisión & Bibliographic \\
Bibliográfica & Review
\end{tabular}

DOI: https://doi.org/10.18537/RFCM.38.03.10

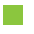

Correspondencia:

luis.suin@ucuenca.edu.ec

Dirección:

Gertrudis Esparza y Vicente

Mideros

Código Postal:

010114

Teléfono:

$2400646-0999763765$

Cuenca - Ecuador

\section{Análisis Envolvente de Datos (DEA) para el estudio de la eficiencia técnica en los Sistemas de Salud: una revisión bibliográfica y metodológica en el contexto ecuatoriano}

\author{
Data Envelopment Analysis (DEA) for the study of technical \\ efficiency in the Health Systems: a bibliographical and \\ methodological review in the Ecuadorian context \\ Suin Guaraca Luis Heriberto¹, Duque Rodríguez Mónica \\ Alexandra², Aguirre Quezada Juan Carlos ${ }^{3}$
}

\section{RESUMEN}

Introducción: el financiamiento de los sistemas de salud ha sido el principal objetivo de las políticas públicas, sobre todo por la utilización de recursos escasos; es necesario que las nuevas intervenciones sanitarias demuestren, además de garantizar su eficacia terapéutica, que son eficientes.

Objetivo: realizar una revisión exhaustiva de la metodología utilizada a propósito de medir la eficiencia técnica en el sector de la salud.

Metodología: se desarrolló una descripción extensa sobre el sistema de salud en el Ecuador y una profunda indagación bibliográfica sobre el DEA, complementando el análisis y síntesis de observaciones particulares y de teorías generales para establecer conclusiones probables y válidas.

Resultados: el DEA se muestra como la metodología que mejor capta la eficiencia en el uso de recursos, fundamentalmente por su versatilidad al incorporar una amplia cantidad de variables de diferente índole y medida, resultando importante por la poca disponibilidad y heterogeneidad de la información existente, calculando y analizando la eficiencia técnica de acuerdo a las necesidades del investigador o a la disponibilidad de datos.

Conclusiones: la investigación describe de manera clara la metodología DEA y particularmente, la importancia y versatilidad que tiene en la medida de la eficiencia técnica en el sector de la salud.

Palabras clave: análisis envolvente de datos, economía de la salud, eficiencia organizacional, sistemas nacionales de salud. Ecuador. 


\section{ABSTRACT}

Introduction: the financing of health systems has been the main objective of public policies, especially due to the use of limited resources; it is necessary that new health interventions demonstrate that they are efficient, in addition to guaranteeing their therapeutic efficacy.

Objective: to carry out an exhaustive review of the methodology used to measure the technical efficiency in the health sector.

Methodology: an extensive description of the health system in Ecuador and a deep bibliographic inquiry on the DEA were developed, complementing the analysis and synthesis of particular observations and general theories to establish probable and valid conclusions.

Results: the DEA is the methodology that best captures the efficiency in the use of resources, fundamentally due to its versatility in incorporating a large number of variables of different nature and measures, being important due to the limited availability and heterogeneity of the existing information, calculating and analyzing technical efficiency according to the researcher's needs or the availability of data.

Conclusions: the research clearly describes the DEA methodology and particularly the importance and versatility it has in the measurement of technical efficiency in the health sector.

Key words: data envelopment analysis, health economics, efficiency organizational, national health systems. Ecuador.

\section{INTRODUCCIÓN}

La salud se define como un estado completo de bienestar físico, mental y social y no solamente como la ausencia de afecciones o enfermedades. Salud para todos contribuirá a mejorar la calidad de vida, la paz, la seguridad, la productividad laboral, la educación, la inversión y el incremento de ingresos ${ }^{1,2}$.

La OMS busca alcanzar la "cobertura universal" en los sistemas de salud, para lo cual es necesario un uso eficiente de los recursos para garantizar la obtención de la cantidad máxima de salud posible, siendo la aplicación fundamental de la economía en el campo sanitario: el análisis de la producción ${ }^{3}$. La economía de la salud trata la forma en que las personas y las organizaciones utilizan recursos escasos para obtener beneficios en salud, tanto en términos de cantidad como de calidad de vida ${ }^{4,5}$ y con la responsabilidad respecto de las necesidades y acceso de la población ${ }^{6}$.

La eficiencia es considerada como una de las condiciones y requisitos para alcanzar el acceso y cobertura universal y la sostenibilidad del sistema sanitario, lo que ha abierto un intenso y progresivo debate, sobre todo en la actualidad, debido a la reciente crisis económica, por lo cual, el presente artículo pretende contribuir con una revisión profunda sobre la metodología utilizada en cuanto a la medición de la eficiencia técnica en el sector de la salud.

\section{Materiales y Métodos}

Se desarrolló un análisis exploratorio de la base legal y operativa para describir el sistema de salud en el Ecuador; y para la metodología, se realizó una exhaustiva indagación bibliográfica 
para conocer los principios básicos, los cambios, los sectores en donde se ha aplicado y los resultados obtenidos en el campo de la salud.

Se han complementado el análisis y la síntesis de los datos y conceptualizaciones, integrando las ideas y favoreciendo la comprensión, además se hizo uso de métodos inductivos y deductivos para lograr razonamientos partiendo tanto desde observaciones particulares como de teorías generales para establecer conclusiones probables y válidas.

Es importante indicar que, al tratarse de entender la base científica del método en cuestión, fue necesario indagar en bibliografía de base, lo que implica que, un porcentaje significativo de referencias, datan de fechas que superan los 5 o 10 años de antigüedad, pero que son totalmente necesarias para el correcto entendimiento de la propuesta realizada.

\section{DESARROLLO}

En el Ecuador la salud es un derecho que constitucionalmente se encuentra garantizado ${ }^{7}$ siendo el Ministerio de Salud Pública (MSP), la autoridad sanitaria nacional que ejerce la rectoría, regulación, planificación y articulación de los actores del sistema nacional de salud y su red pública: MSP, IESS, ISSFA e ISSPOL 8,9 .

El sistema de salud está formado por una parte pública y una privada, cada una dispone de fuentes de financiamiento y orienta sus esfuerzos en atender a cierto sector de la población de características y accesibilidad diferente; en la parte pública resulta importante la labor del Ministerio de Inclusión Económica y Social (MIES) y los servicios de salud que ofertan las municipalidades ${ }^{10}$.
La OPS indica que el acceso y cobertura universal, implican una utilización integral y sin discriminación alguna de los servicios de salud, lamentablemente la región de las Américas no lo ha conseguido, convirtiéndose en una de las más inequitativas en el mundo, debiendo mejorar sus modelos de atención ${ }^{11}$ por lo que, disponer de evidencias sobre la eficacia y seguridad de una alternativa terapéutica no es suficiente para exponer todo su valor, es necesario que las nuevas intervenciones sanitarias demuestren, además de garantizar su eficacia terapéutica, que son eficientes ${ }^{12}$.

La eficiencia medida en función del uso de recursos se convierte en un componente crítico cuando deseamos monitorear y evaluar los sistemas de salud, para ello existen diferentes tipos y metodologías que se utilizan ${ }^{13,14}$, en la tabla $\mathrm{N}^{\circ} 1$ se observan algunas limitaciones y utilidades de las metodologías propuestas.

- Métodos de proporciones simples Análisis de ratios e indicadores

- Métodos de modelos econométricos Análisis de regresión.

- Métodos de frontera - Análisis envolvente de datos 


\section{Tabla $N^{\circ} 1$}

Metodologías para evaluación de los sistemas de salud, sus utilidades y limitaciones

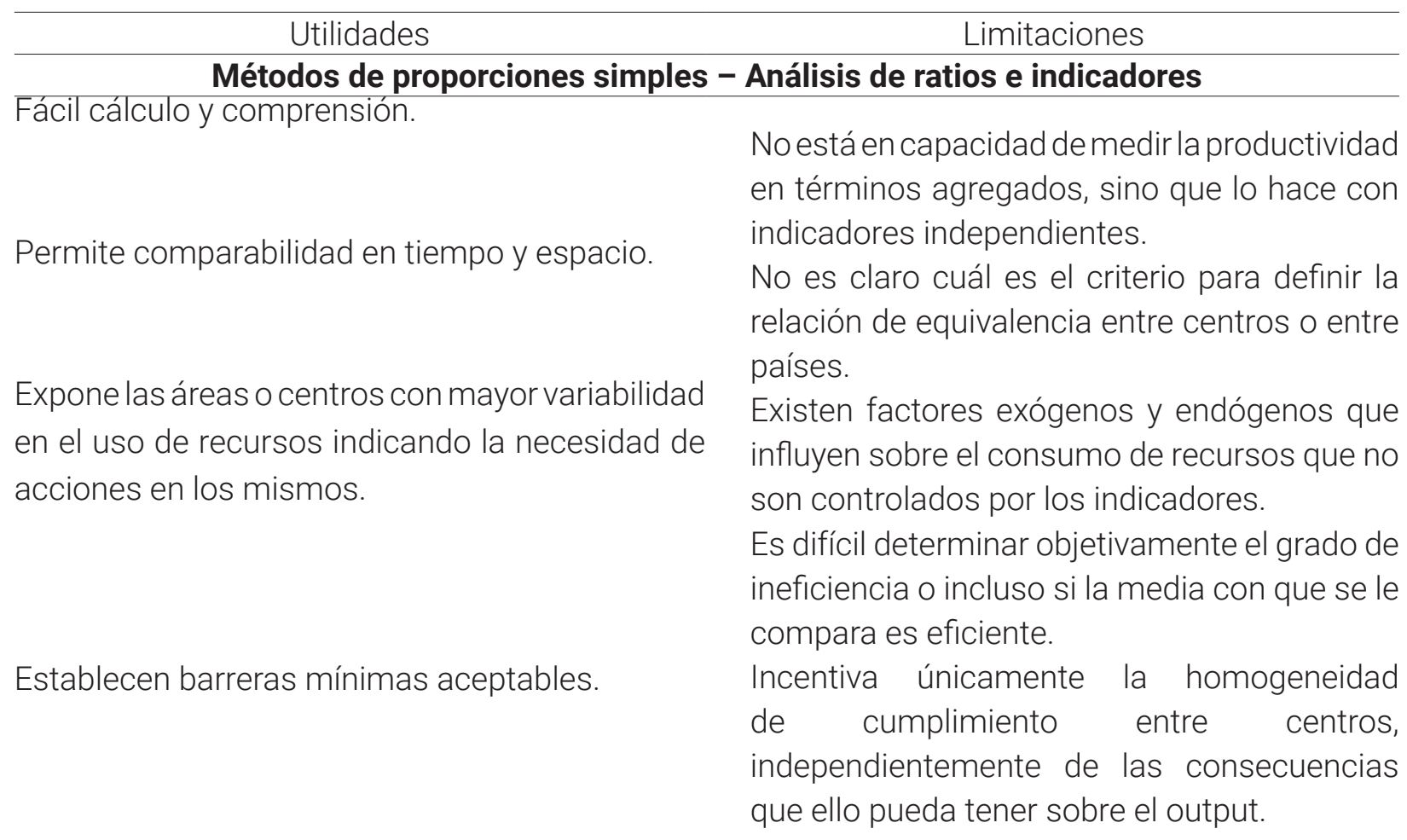

\section{Métodos de modelos econométricos - Análisis de regresión}

Incorpora en el análisis, múltiples inputs y outputs Las estimaciones obtenidas son relaciones de manera simultánea.

Comúnmente aceptada.

medias, pero no necesariamente eficientes,

Contiene indicadores estadísticos para medir el puesto que las observaciones corresponden a término de error (ruido). hospitales eficientes e ineficientes.

Recoge factores endógenos y exógenos que pueden afectar al modelo.

No se puede identificar de forma directa cuales son los hospitales ineficientes, ya que se consideran así, a aquellos que presentan

Posibilidad de aceptar o rechazar hipótesis. costes que distan de la media.

\section{Métodos de frontera - Análisis envolvente de datos}

Flexibilidad en el uso de la información, se adapta a una extensa variedad de inputs y outputs. Asigna en forma matemática pesos óptimos a todos los insumos y productos considerados.

Realiza comparaciones simultáneas de múltiples medidas de desempeño dependientes, calculando eficiencias asignativas y técnicas. Calcula los recursos que pueden ser ahorrados o el producto que debe ser alcanzado. Mide el impacto de las variables no controladas en el desempeño de los centros.
No mide el error.

No mide las diferencias relativas entre los proveedores eficientes.

El uso de muchas variables insumo y producto es considerado metodológicamente erróneo. Requiere de homogeneidad en las unidades utilizadas.

\footnotetext{
Fuente: Peñaloza13; García14; OMS5; Martín¹5; Yates ${ }^{16}$
} 


\section{El Análisis Envolvente de Datos (DEA)}

El DEA es un modelo no paramétrico que se caracteriza por no imponer una forma predeterminada para la función de producción ${ }^{17}$, no existe error de especificación, permite la utilización de múltiples inputs y outputs y admite obtener medidas individuales para cada observación de la muestra utilizada. Farrel $^{18}$ mide la eficiencia productiva en una industria para conocer qué tanto se puede incrementar el producto sin absorber más recursos, dando así el punto de partida a las medidas de eficiencia obtenidas a partir de una metodología de frontera ${ }^{14}$.

Charners ${ }^{19}$ mide la eficiencia técnica relativa de cada industria o unidad de toma de decisiones (DMU); computando el ratio obtenido de dividir la suma ponderada de los outputs y la suma ponderada de los inputs y en función a criterios paretianos, y considerando que el resultado no puede superar la unidad, se construye una frontera de producción empírica para calcular la distancia que existe hacia cada DMU observada; la eficiencia no captada no sigue ningún tipo de distribución probabilística ${ }^{20,21}$.

La figura $N^{\circ} 1$ muestra la curva isocuanta (SS') de una DMU eficiente; si otra DMU utiliza cantidades de insumos definidas por el punto $P$, para producir una unidad de salida, entonces la distancia QP representa la ineficiencia técnica de esa DMU, por lo tanto, la eficiencia técnica (ET) se expresa como la relación ET = 0Q/OP, que es igual a 1 - QP/OP, este resultado toma un valor entre cero y uno como indicador del grado de eficiencia técnica de una DMU ${ }^{22,23}$.

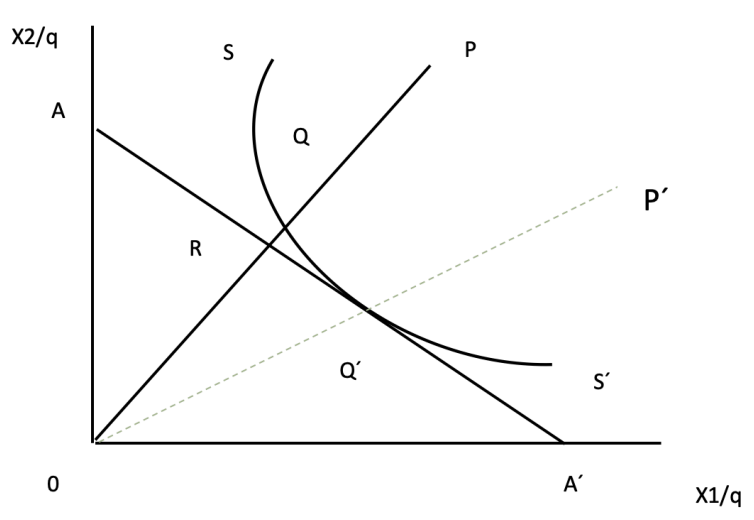

Figura $\mathrm{N}^{\circ} 1$

Representación de la eficiencia técnica y de asignación. Fuente: Emrouznejad et al. ${ }^{23}$; Coelli et al. ${ }^{22}$

Obsérvese que el punto Q es técnicamente eficiente porque se encuentra en la frontera eficiente, en cuyo caso $E T=1$, mientras que la eficiencia de asignación puede calcularse si también se conoce la relación de precios de entrada, representada por la recta presupuestaria AA'. La eficiencia atributiva (EA) se expresa como la relación $E A=0 R / 0 Q$. El punto Q' en donde se intersecan P-A-S, nos muestra tanto ET como EA.

Charnes et al. ${ }^{24}$ propusieron un modelo fraccional básico que relaciona sumas ponderadas de inputs y de outputs, optimizándolos de manera lineal, este método fue conocido como DEA que supone tecnologías con rendimientos constantes a escala - DEA CRS Model - cuya expresión matemática es:

$$
\operatorname{Max}_{u, v} h_{o}=\frac{\sum_{r} U_{r} * Y_{r j 0}}{\sum_{i} V_{i} * X_{i j 0}}
$$

S.A.:

$$
\begin{array}{ccc}
\frac{\sum_{r} U_{r} * Y_{r j 0}}{\sum_{i} V_{i} * X_{i j 0}} \leq 1 & \forall j: 1 \ldots n \\
U r, V i \geq 0 & \forall r: 1 \ldots s & \forall i: 1 \ldots m
\end{array}
$$


Donde:

\section{$h_{o}$ : Función objetivo. Medida de la eficiencia}

Yrj: output $i$ - ésimo de la DMU $j$ - ésima

$X i j$ : input $i$ - ésimo de la DMU $j$ - ésima

Vi, Ur: ponderaciones de inputs y outputs respectivamente

El modelo al ser linealizado, da como resultado dos versiones para maximizar el cociente $h_{0}$, ya sea minimizando la cantidad de inputs para obtener el mismo output (orientación al input) o lograr el máximo output manteniendo los inputs dados (orientación al output), sus expresiones matemáticas son:

$$
\begin{aligned}
& \text { Modelo con orientación input } \\
& h o=\operatorname{Min}_{U_{r}, V_{i}} \sum_{i} V_{i} X_{i j 0} \\
& \text { S.A.: } \\
& \sum_{r} U_{r} Y_{r j}-\sum_{i} V_{i} X_{i j} \leq 0 \quad ; \forall j \\
& \sum_{r} U_{r} Y_{r j 0}=1 \\
& U_{r}, V_{i} \geq 0 \quad ; \forall r, \forall i \\
& h_{0}=\operatorname{Max}_{U_{r}, V_{i}} \sum_{i} U_{r} Y_{r j 0} \\
& \text { S.A.: } \\
& \sum_{r} U_{r} Y_{r j}-\sum_{i} V_{i} X_{i j} \leq 0 \quad ; \forall j \\
& \sum_{r} V_{i} X_{i j 0}=1 \\
& U_{r,}, V_{i} \geq 0 \quad ; \forall r, \forall i
\end{aligned}
$$

\begin{tabular}{|c|c|c|c|}
\hline \multicolumn{2}{|c|}{ Modelo Dual con orientación input } & \multicolumn{2}{|c|}{ Modelo Dual con orientación output } \\
\hline $\operatorname{Min}_{\lambda, h, s_{i}^{-}, s_{r}^{+}} \emptyset$ & & $\operatorname{Max}_{\lambda, h, s_{i}^{-}, s_{r}^{+}} h$ & \\
\hline S.A.: & & S.A.: & \\
\hline$\sum_{j} \lambda_{j} X_{i j}+S_{i}^{-}=\emptyset X_{i j 0}$ & $\forall i$ & $\sum \lambda_{j} X_{i j}+S_{i}^{-}=X_{i j 0}$ & $\forall i$ \\
\hline$\sum \lambda_{j} X_{r j}-S_{r}^{+}=Y_{r j 0}$ & $\forall r$ & $\sum \lambda_{j} X_{r j}-S_{r}^{+}=h Y_{r j 0}$ & $\forall r$ \\
\hline$\psi_{j}$ & & $S_{i}^{+}, S_{r}^{-} \geq 0$ & $\forall i, \forall j$ \\
\hline$S_{i}^{+}, S_{r}^{-} \geq 0$ & $\forall i, \forall j$ & $\lambda_{j} \geq 0$ & $\forall j$ \\
\hline$\lambda_{j} \geq 0$ & $\forall j$ & & \\
\hline
\end{tabular}

Banker et a/25 proponen modelos duales que miden la eficiencia con referencia a los conjuntos de posibilidades de producción y evalúan la eficiencia de cada DMU y cuyas expresiones matemáticas son las siguientes:

Los modelos duales expresan que tanto para h y para $\varnothing$ al alcanzar su óptimo $\left(h^{\star}, \varnothing^{*}=\right.$ 1), no existe ninguna otra combinación, Pareto eficiente, que produzca mayor output o utilice menor input en la producción, proponen además la forma matemática de modelos DEA con rendimientos variables a escala, que básicamente añade una restricción de convexidad, su representación gráfica se puede observar en la Figura № 2.

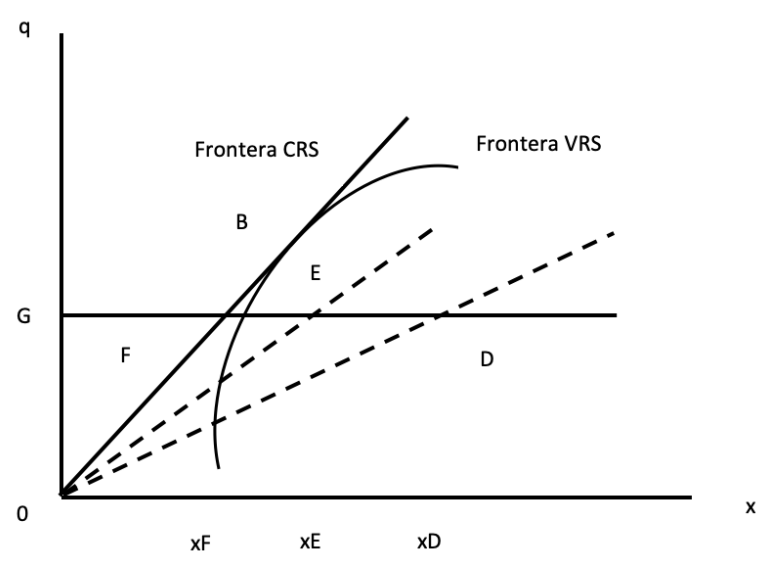

Figura $\mathrm{N}^{\circ} 2$

Representación de la eficiencia técnica y de escala. Fuente: Banker et al. ${ }^{25}$

Las expresiones matemáticas para rendimientos variables a escala son:

\begin{tabular}{llll}
\multicolumn{1}{l}{$\operatorname{Min}_{\lambda, h, s_{i}, s_{r}^{+}} \varnothing$ (6) } & \multicolumn{2}{c}{$\operatorname{Max}_{\lambda, h, s_{i}, s_{r}^{+}} h(7)$} & \\
S.A.: & & S.A.: & \\
$\sum_{j} \lambda_{j} X_{i j}+S_{i}^{+}=\varnothing X_{i j 0}$ & $\forall i$ & $\sum_{\lambda_{j} X_{i j}+S_{i}^{+}=X_{i j 0}}$ & $\forall i$ \\
$\sum_{j} \lambda_{j} X_{r j}-S_{r}^{-}=Y_{r j 0}$ & $\forall r$ & $\sum_{\lambda_{j} X_{r j}-S_{r}^{-}=h Y_{r j 0}}$ & $\forall r$ \\
$\sum_{j} \lambda_{j}=1$ & & $\sum_{j} \lambda_{j}=1$ & \\
$S_{i}^{+}, S_{r}^{-} \geq 0$ & & $S_{i}^{+}, S_{r}^{-} \geq 0$ & $\forall i, \forall j$ \\
$\lambda_{j} \geq 0$ & $\forall i, \forall j$ & $\lambda_{j} \geq 0$ & $\forall j$
\end{tabular}

\section{Aplicación del DEA para medir la eficiencia técnica}

Esta metodología ha sido aplicada en varios sectores, en la educación se demuestra que los recursos son susceptibles de ser mejorados en el uso eficiente de los mismos ${ }^{26,15}$. En el sector económico ecuatoriano, se ha encontrado ineficiencias y una productividad volátil en la mayoría de cooperativas de ahorro y crédito y en pequeñas y medianas empresas (PYMES) 27,28.

En el sector de la salud, Barahona ${ }^{29}$ analiza la eficiencia técnica hospitalaria en Chile, utiliza datos de actividad hospitalaria y personal facultativo en el período 1997-2006 en las 12 regiones del país, encontrando que solamente cuatro regiones son técnicamente eficientes. 
Perez et al. ${ }^{30}$ encuentran un índice medio de eficiencia técnica global (ETG) de 0.736 con una importante variabilidad del $23 \%$ en comunidades autónomas dentro del Sistema Nacional de Salud (SNS) español para el período 2010-2012. Para Fontalvo et al. ${ }^{31}$, existe un exceso en los costos y gastos de venta en 11 de las 18 entidades promotoras de salud (EPS), que fueron analizadas en el período 2010-2012.

Sanmartín et al. ${ }^{32}$ cuantifican la eficiencia relativa del gasto total en salud de sesenta y dos países de América Latina y el Caribe (ALC) y de la Organización para la Cooperación y el Desarrollo Económico (OCDE) en los años 1995, 2005 y 2014, encontrando que el promedio de la eficiencia de los países de ALC fue de 0.938\% y la de los países la OCDE fue de $0.974 \%$. Gómez et al. ${ }^{33}$, afirman que 28 países de la Unión Europea durante el período 2012-2015, han experimentado un pequeño crecimiento de la productividad en los sistemas de salud que se asocia a un cambio positivo en la eficiencia, pero no a mejoras en la tecnología.

Por su parte, Portillo et al. ${ }^{34}$ explican que, en promedio, el $38.27 \%$ de los hospitales públicos del departamento de Nariño entre los años 2008-2014 funcionan de manera eficiente, siendo la demanda potencial, la cartera hospitalaria y la capacidad instalada lo que explica las variaciones de las mediciones obtenidas. Pinzón ${ }^{35}$ determina que, los 203 hospitales de baja complejidad analizados durante el año 2011, deberían reducir las horas de su personal administrativo en un $43.8 \%$, las horas de su personal auxiliar asistencial en un $49.8 \%$, las horas de los profesionales de la salud en un $37.4 \%$ y el $40 \%$ en el número de camas.

Finalmente, el BID $^{36}$ mide los niveles de eficiencia de los sistemas de salud de América Latina y el Caribe, realizando el primer análisis transnacional de la eficiencia del sistema de salud para la región, comparándolos con otros países de ingreso medio y los países de la OCDE, encontrando grandes variaciones en términos de eficiencia, siendo Chile el país mejor posicionado (octavo lugar), compartiendo con la mayoría de países de la OCDE en el 25\% superior, mientras que 22 de los 27 países de la región, se sitúan en la mitad inferior de las clasificaciones de eficiencia promedio. Los países con peor desempeño son Bolivia, Ecuador, Guatemala, Guyana, Panamá y Suriname.

\section{CONCLUSIONES}

La utilización y manejo de los recursos públicos adquiere una dimensionalidad estratégica dentro de la política pública debido a su escasez y el deber de destinarlos para satisfacer necesidades y lograr una mayor utilidad social, convirtiéndose en una asignatura pendiente para los gobiernos de turno, sobre todo en América Latina caracterizada por ser la región más inequitativa del mundo en el campo de la salud.

En ese marco, para que el decidor político tome decisiones enfocadas en maximizar el beneficio social, los recursos limitados deben ser utilizados bajo parámetros de eficiencia si es que se pretende alcanzar una cobertura universal que permitirá, además, mejorar la productividad laboral incrementando los ingresos personales y nacionales.

Sin embargo, la cobertura universal viene siendo un objetivo que no se logra alcanzar, debido, además de otros factores, al uso ineficiente de los recursos; es necesario enfocarnos en medir la correcta utilización del capital en áreas ineficientes específicas y que se asocian con el recurso humano que labora en el sector, la utilización y precio de medicamentos y en los servicios que se prestan; ineficiencias que consumen entre el $20 \%$ y el $40 \%$ de los recursos asignados al campo de la salud. 
Organizaciones internacionales como la OPS y la OMS, proponen una serie de indicadores que son útiles como tasas meta a alcanzar en los diferentes países o regiones, así como referencia para la asignación de recursos a fin de incrementar la atención en salud, sin embargo, no necesariamente nos indican si estos recursos son utilizados de manera eficiente o si su productividad es la máxima que se puede obtener, es decir, estos indicadores se orientan en medir la efectividad y no la eficiencia, pues su metodología se basa en evaluar los resultados.

Se trata entonces de encontrar una metodología que además de garantizar los resultados sanitarios, tenga interés en los recursos empleados y que su utilización sea, definitivamente, la que mejor capte la eficiencia en el uso de los mismos; esta evaluación se la realiza mediante el uso de modelos de regresión o a través del análisis envolvente de datos, siendo este último, el que presentaría cierta ventaja al poder incorporar una amplia cantidad de variables en el análisis (tanto inputs como outputs) y la posibilidad de lidiar con la poca disponibilidad y heterogeneidad de la información existente (que en casos como los de Ecuador, su recolección y presentación es incipiente); permitiendo calcular y analizar la eficiencia técnica desde diferentes ópticas, de acuerdo a las necesidades del investigador o de acuerdo a la disponibilidad de datos.

Dentro de este marco, la investigación alcanza a esbozar de manera clara la metodología DEA y el sistema de salud, y particularmente, la importancia que tiene para los gobiernos y para los responsables de las políticas públicas la utilización y destino de los recursos.

Resulta importante recaudar dinero y destinarlo hacia el servicio de salud, que deberá ser efectivo y además eficiente, para tratar de alcanzar una cobertura universal, contribuir a vidas más sanas, un acceso sin restricciones financieras para la población, y todo esto sin comprometer recursos adicionales que son escasos.

A pesar del esfuerzo de organismos internacionales, la medición de la eficiencia sigue siendo un problema que enfrentar, no existe disponibilidad de datos ni homogeneidad en la información, dificultando la utilización de metodologías y variables adecuadas para medir de manera fehaciente, la eficiencia en el uso de recursos.

A nivel internacional, se ha logrado implementar una serie de indicadores que determinan umbrales mínimos o metas para que los servicios de salud correspondan con efectividad en atención a la sociedad y que están medidos en base a un resultado y no a insumos utilizados.

Bajo estas condiciones, los resultados indican que la metodología que mejor se adapta sería el DEA, pues presenta una flexibilidad en el uso de variables tanto de inputs como de outputs, realizando comparaciones simultáneas y calculando la eficiencia técnica y asignativa en función de recursos ahorrados y producto alcanzado.

Respecto a la revisión bibliográfica, varias investigaciones indican una predominación del uso de esta técnica, sobre todo en América Latina y no solamente en el campo de la salud sino en el análisis de distintos servicios públicos, así como también en el sector privado y financiero.

Finalmente, esta investigación contribuye con un acercamiento a la medición de la eficiencia en el campo de la salud considerando la metodología DEA, la cual resulta importante como base para futuras investigaciones en el campo de la economía de la salud. 


\section{INFORMACIÓN DE LOS AUTORES}

Suin Guaraca Luis Heriberto. Economista. Magister en Administración de Empresas. Libre ejercicio. Cuenca - Azuay - Ecuador.

e-mail: luis.suin@ucuenca.edu.ec

ORCID:https://orcid.org/0000-0003-3465-2835

Duque Rodríguez Mónica Alexandra. Ingeniera en Contabilidad y Auditoría. Magister en Contabilidad y Finanzas con Mención en Gerencia y Planeamiento Tributario. Universidad de Cuenca. Facultad de Ciencias Económicas y Administrativas - FCEA. Carrera de Administración de Empresas Dual. Cuenca Azuay - Ecuador.

e-mail: monica.duque@ucuenca.edu.ec

ORCID:https://orcid.org/0000-0003-4315-2584

Aguirre Quezada Juan Carlos. Ingeniero Financiero. Doctor dentro del Programa de Doctorado en Ciencias Jurídicas y Económicas. Universidad de Cuenca. Facultad de Ciencias Económicas y Administrativas - FCEA. Carrera de Administración de Empresas. Azogues Cañar - Ecuador.

e-mail: juan.aguirreq@ucuenca.edu.ec ORCID:https://orcid.org/0000-0002-8953-8151

\section{CONTRIBUCIÓN DE LOS AUTORES}

Todos los autores contribuyeron de manera similar en la concepción y diseño del trabajo, análisis e interpretación de los datos, redacción y revisión crítica del manuscrito y aprobación de la versión final.

\section{CONFLICTO DE INTERESES}

Los autores declaran la no existencia de conflicto de intereses.

\section{FUENTES DE FINANCIAMIENTO}

Autofinanciado

\section{REFERENCIAS BIBLIOGRÁFICAS}

1 OMS (Organización Mundial de la Salud). La financiación de los sistemas de salud: el camino hacia la cobertura universal. Informe sobre la salud en el mundo [Internet]. 2010 [citado 5 de marzo 2020]. Disponible en: https://www.who.int/ whr/2010/10_summary_es.pdf?ua=1

2 Jamison D, Summers L, Alleyne G, Arrow K, Berkley S, Binagwaho A, et al. Salud global 2035: un mundo convergiendo en el lapso de una generación. Salud Pública de México [Internet]. 2015 oct [citado 7 de mayo 2020]; 57(5): 444-7. Disponible en: https://www.medigraphic.com/pdfs/ salpubmex/sal-2015/sal155l.pdf

3 Ortún V, Meneu de Guillerna R. Impacto de la economía en la política y gestión sanitaria. Rev Esp Salud Pública [Internet]. 2006 oct [citado 13 de febrero 2020]; 80(5): 491-4. Disponible en: http:// scielo.isciii.es/scielo.php?script =sci_ arttext\&pid=\$1135-57272006000500008

4 López G, Ortún V. Economía y salud: fundamentos y políticas. 2da ed. Madrid: Encuentro; 1998.

5 OMS (Organización Mundial de la Salud). Las 10 principales causas de defunción [Internet]. 2019 dic [citado 15 de marzo 2020]. Disponible en: https://www.who.int/ es/news-room/fact-sheets/detail/the-top10-causes-of-death

6 Cid C, Báscolo E, Morales C. La eficiencia en la agenda de la estrategia de acceso y cobertura universales en salud en las Américas. Salud Pública de México [Internet]. 2016 sep 26 [citado 3 de febrero 2020]: 58(5): 496-3. Doi: http://dx.doi. org/10.21149/spm.v58i5.8182 
7 Constitución de la República del Ecuador. Registro Oficial, Suplemento 449. Quito, Ecuador: Asamblea Nacional [Internet]. (Oct 20, 2008). [citado 4 de abril 2020]. Disponible en: https://www.oas.org/ juridico/pdfs/mesicic4_ecu_const.pdf

8 Reglamento del Código Orgánico de Planificación y Finanzas Públicas. Registro Oficial, Suplemento 383. Quito, Ecuador. Presidencia de la República del Ecuador [Internet]. (Nov 26, 2014). [citado 17 de mayo 2020]. Disponible en: https://www. planificacion.gob.ec/wp-content/uploads/ downloads/2018/09/Reglamento-CodigoOrganico-de-Planificaci\%C3\%B3n-yFinanzas-Publcias.pdf

9 MSP (Ministerio de Salud Pública). Convenio marco interinstitucional entre el Ministerio del Interior, Ministerio de Defensa, IESS, ISSFA, ISSPOL y Ministerio de Salud Pública para integra la Red Pública Integral de Salud [Internet]. (Abr 10, 2015). [citado 2 de mayo 2020]. Disponible en: $\quad$ http://instituciones.msp.gob.ec/ images/Documentos/subse_gobernanza/ Convenio_RPIS\%202015.pdf

10 Lucio R, Nilhda V, Henriquez R. Sistemas de Salud en Ecuador. Salud Pública de México [Internet], 2011 [citado 22 de mayo 2020] 53: 177-7. Disponible en: https://www. redalyc.org/pdf/106/10619779013.pdf

11 OPS (Organización Panamericana de la Salud). Estrategia para el acceso universal a la salud y la cobertura universal de salud: hacia el consenso en la región de las Américas [Internet]. 2014 [citado 13 de abril 2020]. Disponible en: https://www. paho.org/hq/dmdocuments/2014/UHCconsultas-CD53-Spanish-2014.pdf?ua=1
12 Soto J, Casado M. La trayectoria metodológica de la evaluación de la eficiencia y su futuro. Papeles de Economía Española [Internet]. 2019 [citado 8 de marzo 2020]; 160: 156-4. Disponible en: https://www.funcas.es/wp-content/ uploads/Migracion/Articulos/FUNCAS_ PEE/160art15.pdf

13 Peñaloza M. Evaluación de la Eficiencia en instituciones hospitalarias públicas y privadas con Data Envelopment Analysis (DEA). Archivos de Economía [Internet]. 2003 dic [citado 18 de febrero 2020]; 1-3. Disponible en: https://www.researchgate. net/publication/5007267_Evaluacion_ de_la_Eficiencia_en_Instituciones_ Hospitalarias_publicas_y_privadas_con_ Data_Envelopment_Analysis_DEA

14 García B. Análisis de eficiencia del sector hospitalario: una revisión de métodos. Cuaderno de estudios empresariales [Internet]. 1997 jun [citado 23 de febrero 2020]; (7): 151-6. Disponible en: https:// www.researchgate.net/profile/BeatrizGarcia-Cornejo/publication/27581587_ Analisis_de_eficiencia_del_sector_ hospitalario_una_revision_de_metodos/ links/54f874ea0cf2ccffe9df3013/Analisisde-eficiencia-del-sector-hospitalario-unarevision-de-metodos.pdf

15 Martín R. La medición de la eficiencia universitaria: una aplicación del Análisis Envolvente de Datos. Form Univ [Internet]. 2008 [citado 15 de marzo 2020]; 1(2): 176. Doi: http://dx.doi.org/10.4067/S071850062008000200004

16 Yates J. When Will Players Get Involved?. Health Social Services Journal. 1983: (15): 1101-2. 
17 FÖrsund F, Lovell K, Schmitd P. A survey of frontier production functions and of their relationship to efficiency measurement. Journal of Econometrics [Internet]. 1980 may [citado 29 de mayo 2020]; (13): 5-5. Doi: https://doi.org/10.1016/03044076(80)90040-8

18 Farrel M. The measurement of productive efficiency. Journal of the Royal Statistical Society [Internet]. 1957 mar 20 [citado 22 de abril 2020]; 120(3): 253-0. Doi: https:// doi.org/10.2307/2343100

19 Charnes A. Data Envelopment Analysis: Theory, Methodology and Applications, 2da ed. New York: Kluwer Academic Publishers; 1997.

20 Hannoch G, Rothschild M. Testing the assumptions of production theory: a nonparametric approach. Journal of Political Economy [Internet]. 1972 [citado 19 de abril 2020]; 80(2): 256-5. Doi: https://www.journals.uchicago.edu/doi/ pdf/10.1086/259881

21 Sengupta J. Efficiency Measurement in Non Market Systems Thoroung DEA. International Journal of Systems Science [Internet]. 1987 abr: 18 [citado 23 de marzo 2020]; 2279-4. Doi: https://doi. org/10.1080/00207728708967187

22 Coelli T, Rao D, O’Donnell C, Battese G. An Introduction to Efficiency and Productivity Analysis. 2da ed. New York: Springer Science; 2005.

23 Emrouznejad A, Cabanda, E. Introduction to Data Envelopment Analysis and its applications, inOsmanetal.(Eds.)Handbook of Research on Strategic Performance Management and Measurement Using Data Envelopment Analysis. IGI Global
[Internet]. 2015 [citado 15 de mayo 2020]; 235-5. Disponible en: https://deazone. com/en/emrouznejad-a-and-e-cabanda2015-introduction-to-data-envelopmentanalysis-and-its-applications-in-osmanet-al-eds-handbook-of-research-onstrategic-performance-management-andmeasurement

24 Charnes A, Cooper W, Rhodes E. Measuring the efficiency of decision making units. European Journal of Operations Research [Internet]. 1978 [citado 25 de abril 2020]; 2(6): 429-4. Disponible en: https://personal. utdallas.edu/ ryoung/phdseminar/ CCR1978.pdf

25 Banker R, Charnes A, Cooper W. Some models for estimating technical and scale inefficiencies in data envelopment analysis. Management Science [Internet]. 1984 [citado 11 de abril 2020]; 30: 10782. Disponible en: http://deazone.com/ en/banker-rd-a-charnes-and-ww-cooper1984-some-models-for-estimatingtechnical-and-scale-inefficiencies-in-dataenvelopment-analysis-management-science-309-1078-1092

26 Villarreal F, Tohmé F. Análisis envolvente de datos. Un caso de estudio para una universidad argentina. Estudios Gerenciales [Internet]. 2017 sep [citado 14 de abril 2020]; 33: 302-8. Doi: https://doi. org/10.1016/j.estger.2017.06.004

27 Campoverde J, Romero C, Borestein D. Evaluation of the efficiency of savings and credit cooperatives in Ecuador: A Data Envelopment Analysis (DEA) aplication. Contaduría y Administración [Internet]. 2018 nov [citado 3 de mayo 2020]; 64(1): 1-9. Doi: http://dx.doi.org/10.22201/ fca.24488410e.2018.1449 
28 Pumisacho V, Alvarado K. Evaluación de eficiencia y productividad de PyMEs productivas usando análisis envolvente de datos e índice Malmquist. Espacios [Internet]. 2018 abr 12 [citado 22 de mayo 2020]; 39(33): 10-7. Disponible en: http:// www.revistaespacios.com/a18v39n33/ a18v39n33p10.pdf

29 Barahona P. Análisis de eficiencia hospitalaria en Chile. An Fac med [Internet]. 2011 [citado 12 de marzo 2020]; 72(1), 338. Disponible en: https://www.redalyc.org/ pdf/379/37920884006.pdf

30 Pérez C, Ortega M, Ocaña R, Martín J. Análisis de la eficiencia técnica en los hospitales del Sistema Nacional de Salud español. Gac Sanit [Internet]. 2017 abr [citado 25 de marzo 2020]; 31(2): 108-5. Doi: http://dx.doi.org/10.1016/j. gaceta.2016.10.007

31 Fontalvo T, Adel M, Delimiro V. Medición de la eficiencia financiera de las entidades promotoras de salud (eps) del régimen contributivo mediante el análisis envolvente de datos (aed). Universidad \& Empresa [Internet]. 2015 dic [citado 22 de febrero 2020]; 17(29): 93-0. Doi: http://dx.doi.org/10.12804/rev.univ. empresa.29.2015.04

32 Sanmartín D, Henao $M$, Valencia $Y$, Restrepo J. Eficiencia del gasto en salud en la OCDE y ALC: un análisis envolvente de datos. Lecturas de Economía [Internet]. 2019 jul 17 [citado 23 de febrero 2020]; 91 : 41-8. Doi: https://doi.org/10.17533/udea. le.n91a02

33 Gómez J, García J, Gómez M. Eficiencia y productividad de los sistemas de salud en los países de la Unión Europea. Estudios de Economía Aplicada [Internet]. 2019 [citado 12 de mayo 2020]; 37(2): 196-5. Disponible en: https://dialnet.unirioja.es/ servlet/articulo?codigo $=6963183$
34 Portillo D, Enríquez F, Riascos J. Factores económicos que inciden sobre el índice de eficiencia técnica de los hospitales públicos del departamento de Nariño - Colombia 2008-2014. Apuntes del Cenes [Internet]. 2019 feb 07 [citado 8 de abril 2020]; 38(67): 193-5. Doi: https://doi.org/10.19053/01203053.v38. n67.2019.7364

35 Pinzón M. Medición de eficiencia técnica relativa en hospitales públicos de baja complejidad mediante la metodología Data Envelopment Analysis (DEA) [tesis de maestría]. Pontificia Universidad Javeriana, Colombia [Internet]. (2003) [citado 21 de abril 2020]. Disponible en: https://colaboracion.dnp.gov.co/CDT/ Estudios\%20Econmicos/245.pdf

36 BID (Banco Interamericano de Desarrollo). Mejor gasto para mejores vidas: cómo América Latina y el Caribe puede hacer más con menos. Informe de Desarrollo en las Américas [Internet]. 2018 [citado 12 de febrero 2020]. Disponible en: https:// publications.iadb.org/publications/ spanish/document/Resumen_ejecutivo_-DIA_2018_-_Mejor_gasto_para_mejores_ vidas_-_C_mo_Am_rica_Latina_y_Caribe_ puede_hacer_m_s_con_menos.pdf 